\title{
Determinant Factors of the Direct Medical Costs Associated with Genotype 1 Hepatitis C Infection in Treatment-Experienced Patients
}

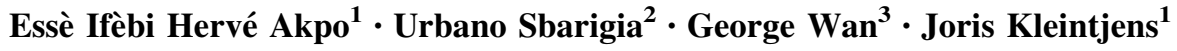

Published online: 28 September 2015

(C) The Author(s) 2015. This article is published with open access at Springerlink.com

\begin{abstract}
Objective Limited evidence is available on predictors of medical resource utilization (MRU) and related direct costs, especially in treatment-experienced patients infected with genotype 1 hepatitis $\mathrm{C}$ virus (HCV). This study aimed at investigating patient and treatment characteristics that
\end{abstract}

Electronic supplementary material The online version of this article (doi:10.1007/s40268-015-0109-5) contains supplementary material, which is available to authorized users.

Essè Ifèbi Hervé Akpo

eakpo@deloitte.com

Deloitte, Berkenlaan 8a, 1831 Diegem, Belgium

Janssen Pharmaceutica NV, Beerse, Belgium

3 Janssen Global Services LLC, Raritan, NJ, USA predict MRU and related non-drug costs in treatment-experienced patients with chronic hepatitis $\mathrm{C}(\mathrm{CHC})$ treated with simeprevir (SMV) or telapravir (TVR) in combination with pegylated interferon and ribavirin (PegIFN/R).

Patients and Methods A total of 709 patients who completed the 72-week ATTAIN trial were included in the study. Cost data were analysed from the UK NHS perspective. Descriptive statistics and regression analyses were used to determine patterns and predictors of total MRU-related costs associated with SMV/PegIFN/R and TVR/PegIFN/R.

Results Independent predictors for total MRU-related costs were age, region and the following interaction terms: (1) gender $\times$ F3-F4 METAVIR score $\times$ baseline viral load (BLVL), (2) body mass index $(\mathrm{BMI}) \times \mathrm{F} 3-\mathrm{F} 4$ METAVIR score $\times$ prior response to PegIFN/R and (3) gender $\times$ achievement of SVR at 12 weeks $($ SVR12) $\times$ BLVL. A F3-F4 METAVIR score was a stronger predictor of total MRU-related costs than SVR12. Predictors of adverse events included older age, female gender, low BMI, TVR/PegIFN/R and SVR12. Wilcoxon rank sum test revealed comparable total MRU-related costs between SMV/PegIFN/R and TVR/PegIFN/R.

Conclusion To the best of our knowledge, this study is the first to describe the relationship between commonly admitted predictors of MRU-related costs and their joint effect on total MRU-related costs in treatment-experienced patients with CHC. The identified predictors of MRU-related costs suggest that significant treatment costs can be avoided by starting treatment early before the disease progresses. Furthermore, adverse events seem to be the most important factor to take into consideration for the choice of treatment, especially when therapeutic options are associated with similar levels of medical resource utilization and associated costs. 


\section{Key Points}

Early age identification and treatment of patients with hepatitis $\mathrm{C}$ infection would limit non-response and advanced liver disease and associated non-drugrelated costs. A weight-loss programme should further be considered in the management of treatment-experienced patients with chronic hepatitis C.

The achievement of a sustained virologic response at week 12 is associated with significant reduction in non-drug-related costs, an effect that is strongly dependent on both liver disease severity and baseline viral load. This study advocates for a close monitoring of liver disease fibrosis together with (and not independently from) baseline viral load when caring for treatment-experienced patients with chronic hepatitis $\mathrm{C}$.

Finally, in clinical practice, the choice of treatment for experienced patients with chronic hepatitis $\mathrm{C}$ should be guided primarily by the safety profile and the risk of adverse events of the direct-acting antiviral agents.

\section{Introduction}

Hepatitis $\mathrm{C}$ virus (HCV) is the leading cause of chronic hepatitis $\mathrm{C}(\mathrm{CHC})$, liver cirrhosis, hepatocellular carcinoma and liver transplantation $[1,2]$. HCV infection accounts for $33 \%$ and $20 \%$ of total liver cancer in developing and developed countries, respectively [3]. Hence, CHC is acknowledged as a major public health problem. The disease burden of $\mathrm{HCV}$ in Europe is currently at its peak given the prolonged time-course of the condition-individuals that were infected in the 1980s (when the disease propagation outbreak was significant) are currently developing cirrhosis $[4,5]$. The clinical management requires efficacious drugs that stop the progression of the disease and reduce the number of $\mathrm{HCV}$-infected patients that could potentially develop complications. Consequently, the limitations of the dual therapy pegylated interferon plus ribavirin (PegIFN/R, former standard of care) and of the first generation of directacting agents [telaprevir (TVR) and boceprevir (BCP)] prompted the development of new therapies including simeprevir (SMV) with improved tolerability, applicability and compliance profiles as compared to TVR triple therapies (i.e., in combination with PegIFN/R) [6-9]. As new regimens may come at a higher price, decision makers also need to understand patient and treatment characteristics that influence resource use, and thus the total healthcare costs. This is shown by the cost effectiveness of new antiviral drugs that can be further improved by efficient use and savings in medical resources. Although it should be noted that drug costs, in particular direct-acting agents, constitute $80-90 \%$ of the total direct cost [10-13] and about $50 \%$ of the total direct and indirect costs [14] in patients with CHC. Understanding the parameters associated with high healthcare costs may then be useful in improving efficiency in care delivery for patients with high-cost CHC. This is particularly relevant for disease management programmes aiming at improving the welfare of patients with chronic conditions that generally consume more healthcare services, including physician visits, specialist visits and hospital care $[15,16]$.

Backx et al. recently quantified the medium-term difference in medical resource utilization (MRU) and related costs based on response status to PegIFN/R treatment [17]. They reported per patient per year non-drug costs of $£ 806$ and $£ 506$ for retreated and non-retreated patients with $\mathrm{CHC}$, respectively. The additional non-drug-related costs for the retreated patients were mainly attributable to increased numbers of outpatient visits and HCV RNA tests related to treatment monitoring. Consequently, care for patients with $\mathrm{CHC}$ who failed to achieve a sustained virologic response (SVR, a marker of viral clearance and of cure) places a significant economic burden on health services [18]. However, the existing literature poorly characterizes factors that predict high and low MRU and related costs, especially in treatment-experienced patients infected with genotype $1 \mathrm{HCV}$. The purpose of this study was to determine whether a significant relationship exists between baseline demographics, clinical characteristics and treatment features and non-drug MRU-related costs among treatment-experienced patients. Medical resource data collected alongside the ATTAIN pivotal trial (Simeprevir vs Telaprevir with Peginterferon and Ribavirin in Previous Null or Partial Responders with Chronic Hepatitis C Virus Genotype 1 Infection) were used for this purpose. In addition, this study looked at how SMV/PegIFN/R and TVR/PegIFN/R affected MRU and associated costs.

\section{Methods}

\subsection{Study Design}

The ATTAIN trial was a phase III, randomized, doubleblind, non-inferiority trial set up to evaluate the efficacy, safety and tolerability of SMV/PegIFN/R versus TVR/ PegIFN/R triple regimens in CHC genotype 1 infected patients who were prior partial or null responders to PegIFN/R therapy. The study comprised a 48-week 
treatment phase followed by a post-therapy period up to 72 weeks after the start of the treatment. MRU data were collected alongside the trial and included hospitalization days, nights in the intensive care unit as well as emergency room, gastroenterologist, hepatologist, psychiatrist, psychologist, infectious disease specialist, nurse, primary care physician, surgeon, social worker, home and other (physiotherapist) visits.

\subsection{Cost Data}

The UK NHS perspective was considered to estimate MRU-related unit costs. Unit costs for each resource were derived from NHS reference costs [19] or the 'Unit Costs of Health and Social Care' from the Personal Social Services Research Unit (PSSRU) [20, 21]. Costs were inflated to 2014 values using the medical component of the Consumer Price Index [22]. The unit costs are summarized in Table 1. The total MRU-related cost per patient was calculated by multiplying each resource by its unit costs and then summing the individual MRU-related costs for each patient or treatment arm. Thus, this study applies only to service care provision, as costs related to tests, procedures and medications were not analysed.

\subsection{Statistical Analyses}

\subsubsection{Dataset}

The analysis was based on the 72-week study period that included treatment and follow-up phases. For each patient, data on demographics [age, gender, geographical region and baseline body mass index (BMI)], clinical characteristics [baseline viral load (BLVL), IL28B polymorphism, F3-F4 fibrosis score, prior response to PegIFN/R] and treatment features [treatment status (SMV/PegIFN/R vs TVR/PegIFN/R), SVR12 achievement and adverse events] were gathered. With respect to treatment features, adverse events encompassed anaemia, bilirubin, neutropenia, pruritus, rash and absence of any of these adverse events. An additional categorical variable level was created to account for joint occurrences or potential interactions between each of the selected adverse events and the others. Capturing first-order to fourth-order interactions would have entailed an additional 20 covariates, which could result in convergence issues. Patients with missing data and total MRUrelated cost outliers were excluded from the analysis. To identify outliers, a simple ordinary least square (OLS) regression was fitted to total MRU-related costs while controlling for all factors. Total MRU-related costs with
Table 1 Unit cost per medical resource (£, UK tariff)

\begin{tabular}{|c|c|c|c|c|}
\hline & \multicolumn{2}{|c|}{ Unit cost (£) 2011-2012 } & \multicolumn{2}{|c|}{ Inflation correction to 2014} \\
\hline & $\begin{array}{l}\text { First } \\
\text { attendance }\end{array}$ & $\begin{array}{l}\text { Follow-up } \\
\text { attendance }\end{array}$ & $\begin{array}{l}\text { First } \\
\text { attendance }\end{array}$ & $\begin{array}{l}\text { Follow-up } \\
\text { attendance }\end{array}$ \\
\hline Emergency room visit & 122.00 & 33.00 & 130.57 & 35.32 \\
\hline Gastroenterologist visit & 164.00 & 45.00 & 175.52 & 48.16 \\
\hline Hepatologist visit & 216.00 & 27.00 & 231.17 & 28.90 \\
\hline Infectious disease specialist visit & 280.00 & 164.00 & 299.67 & 175.52 \\
\hline Psychiatrist visit & 178.00 & 178.00 & 190.50 & 190.50 \\
\hline Psychologist visit & 56.00 & 157.00 & 59.93 & 168.03 \\
\hline Surgeon visit & 145.00 & 112.00 & 155.18 & 119.87 \\
\hline Home visit & 70.00 & 29.00 & 74.92 & 31.04 \\
\hline Nurse visit & 21.00 & 21.00 & 22.47 & 22.47 \\
\hline Night in intensive care unit & 868.00 & 868.00 & 928.96 & 928.96 \\
\hline Primary care physician visit ${ }^{\mathrm{a}}$ & 54.00 & 54.00 & 63.71 & 63.71 \\
\hline Social worker visit ${ }^{\mathrm{a}}$ & 30.00 & 30.00 & 35.39 & 35.39 \\
\hline Night in hospital ${ }^{\mathrm{b}}$ & 235.00 & 235.00 & 287.30 & 287.30 \\
\hline Other visit $^{\mathrm{c}}$ & 43.00 & - & 46.02 & - \\
\hline
\end{tabular}

Tariff data were taken from National Schedule of Reference Costs 2011-2012 unless stated otherwise

a Curtis L. Unit Costs of Health and Social Care 2008. Personal Social Services Research Unit (PSSRU), University of Kent, 2008

b Curtis L. Unit Costs of Health and Social Care 2007. Personal Social Services Research Unit (PSSRU), University of Kent, 2007

c A conservative estimate of the unit costs for physiotherapy was applied because the majority of 'other visits' were related to physiotherapy and other types of visits in this category were typically more expensive 
studentized statistical residue outside the range -2 to 2 were considered as outliers and removed from the database.

\subsubsection{Data Analysis}

A two-step approach was undertaken to tackle the objective of the study. The first step consisted of determining the relationship between total MRU-related costs and each of the various patient and treatment variables. Analysis of variance and simple OLS regressions were used for categorical and continuous variables, respectively. For those categorical variables that showed significance at the $5 \%$ level, Tukey's HSD (honestly significant difference) post hoc test was used to detect where the difference lies between levels. Multivariable regression was then used in a second step to provide the independent contribution of each of the patient and treatment variables to the prediction of total MRU-related costs. For that, variables with linear effects were used as explanatory variables in a stepwise multiple OLS regression that aimed at determining the strongest and most effective predictors of total MRU-related costs. The rationale behind the use of OLS regression, despite the skewed distribution of total MRU-related cost data, was that inferences could be made about the arithmetic mean; the most informative measures for healthcare policy decision makers [23].

Checks were then performed to validate the results of the multivariable regression analyses, including non-parametric bootstrapping to assess the robustness of confidence intervals and $p$ values to non-normality in the total MRUrelated costs distribution [24]. Additionally, results of the multivariable OLS regression models were systematically compared with those obtained from a stepwise generalized linear model (GLM) where a gamma distribution was assumed for total MRU-related costs. Of note, this validation exercise takes into account only patients with a positive cost [25]. Chi-square statistics were used to validate GLM models, and descriptive statistics and Wilcoxonrank sum test conducted to compare the pattern of MRUrelated costs between SMV/PegIFN/R and TVR/PegIFN/R.

Co-linearity between independent variables was evaluated through the calculation of the variance inflation factor (VIF). A VIF score $>2$ was taken as an indication of multicollinearity in multivariable regressions. $p$ values associated with regression coefficients between $1 \%$ and $5 \%$ were considered at the borderline of statistical significance; an indication of possible interaction with other variables. These potential interactions between variables and the extent to which they were associated with total MRU-related costs were further investigated. Finally, to provide further insight into the interaction effects, a series of stepwise OLS, logistic and multinomial regression analyses was conducted for numeric, two-level and more than two-level categorical variables, respectively. Statistical analyses were carried out in R (http://www.r-project. org/).

\section{Results}

\subsection{Dataset}

The intention-to-treat (ITT) population included 763 prior non-responders to PegIFN/R. Data screening for outliers resulted in excluding 18 data points. The removal of the outliers was deemed appropriate given that the adjusted $R^{2}$ of the two OLS models fitted to the total MRU-related costs (one model including the outliers, the other not) were virtually similar (data not shown). Thirty-six patients with incomplete data were further excluded from the analysis. The final set of 709 patients was then used to compute regression models and descriptive statistics for MRU-related costs. This is equal to $93 \%$ of the ITT population.

\subsection{Total MRU-Related Costs}

For the entire trial population, total MRU-related costs per patient averaged $£ 363.34 \pm$ standard deviation (SD): $£ 746.45$; median: $£ 63.71$; interquartile range (IQR): $£ 318.53$; indicating positive skewness of the costs (Fig. 1). To determine the resources that contributed the most to total MRU-related costs, univariate OLS regression analyses were fitted to total MRU-related costs while controlling for each type of medical resources. The variation in total MRU-related costs was found to be mainly attributable to nights in hospital (63\%, data not shown).

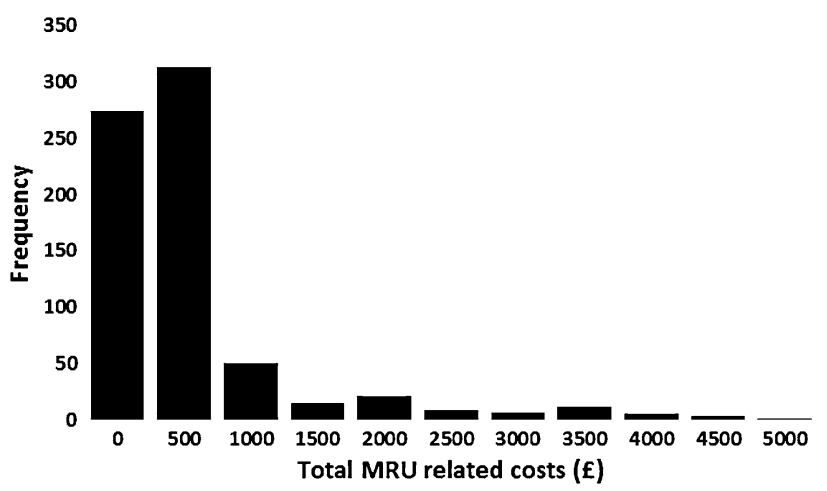

Fig. 1 Histogram of total medical resource utilization (MRU)-related costs $(£)$ for the prior non-responders to pegylated interferon and ribavirin (PegIFN/R) in the ATTAIN trial. The $x$ axis represents the range of non-drug total MRU-related costs, calculated as follows: unit costs for each of the selected resources were multiplied by the number of visits made by each patient. Costs across the medical resources were then summed up for each of the patients. Costs were then grouped into ten bins and frequency calculated accordingly 


\subsection{Univariate Predictors of Total MRU-Related Costs and Interaction Terms}

To identify patient demographics (clinical characteristics and treatment features that can influence total MRU-related costs), univariate analyses were performed. The results from this analysis are shown in Table 2. For patient demographics, higher costs were found to be significantly associated with higher age $(p<0.001)$. Each additional year of age was associated with an average increase of $£ 8.98$ in total MRU-related costs. In addition, differences were found across the various regions $(p<0.05)$. Tukey's HSD analyses revealed higher costs in North America compared with South American regions, with a mean difference of $£ 326.91$ ( $p<0.05$, data not shown). Gender and BMI were found to be at the borderline significance level in their contribution to explaining the variation in costs $(p<0.1)$. For the clinical characteristics, patients with severe liver fibrosis (F3-F4 METAVIR score) incurred higher costs than those with mild to moderate liver fibrosis (F0-F2 METAVIR score), with a mean difference of $£ 198.21$ (Tukey's HSD test, $p<0.001$ ). Finally, for the treatment features, the association between SVR12 and costs was borderline significant.

After identifying interaction effects, the variables that showed borderline significance levels (gender, BMI and SVR12) were further investigated. However, prior to studying these interactions through multivariable regression analyses, tests for multicollinearity were conducted. Results revealed VIF values $<2$, indicating that significant multicollinearity was not present in the selected predictor variables.

For the interactions between gender and any other covariates, multivariable OLS regression with total MRUrelated costs as the dependent variable did not reveal significant first-order interaction effects. However, significant second-order interaction effects were identified between gender $\times$ F3-F4 $\times$ BLVL $(p<0.05)$ (data not shown).

For the interactions between BMI and any other covariates, no significant first-order interaction effects were found, although BMI $\times$ F3-F4 was found to be borderline significant (data not shown). Subsequent examination led to identification of the following three second-order interaction terms: BMI $\times$ F3-F4 $\times$ prior response to PegIFN/R $(p<0.05), \quad$ BMI $\times \mathrm{F} 3-\mathrm{F} 4 \times$ therapy $\quad(p<0.1) \quad$ and $\mathrm{BMI} \times \mathrm{F} 3-\mathrm{F} 4 \times$ region $(p<0.05)$. The model with the interaction term BMI $\times$ F3-F4 $\times$ therapy did not yield to higher predictive power than the one with only the interaction term BMI $\times$ F3-F4. Therefore, the second model was rejected and no third-order interaction was investigated. With the BMI $\times$ F3-F4 $\times$ region model, the relatively large standard error of the coefficient and the small number of the Asian-Pacific patients limited the confidence in this finding and led also to the rejection of this model (data not shown).

For the interactions between SVR12 and any other covariates, the coefficient associated with the second-order interaction term gender $\times$ SVR1 $2 \times$ BLVL was found to be significant at the $5 \%$ level (data not shown).

Overall, the results described above were confirmed by GLM models with a gamma log link function fitted to total MRU-related costs with the same set of predictors. In summary, age, region and the interaction terms gender $\times$ F3-F4 $\times$ BLVL, BMI $\times$ F3-F4 $\times$ prior response to PegIFN/R and gender $\times$ SVR1 $2 \times$ BLVL were significantly associated with total MRU-related costs.

\subsection{Multivariable Predictors of Total MRU-Related Costs}

The independent contribution of the predictors that showed significant univariate correlation to total MRU-related costs was examined next. Stepwise multivariable OLS and GLM regression models were fitted to total MRU-related costs while controlling for the following variables: age, region and the interaction terms gender $\times \mathrm{F} 3-\mathrm{F} 4 \times$ BLVL, BMI $\times$ F3-F4 $\times$ prior response to PegIFN/R and gender $\times$ SVR12 $\times$ BLVL. The coefficients of the following variables and interaction terms were significantly different from zero in the multivariable OLS (Table 3) and GLM (Table S1, see electronic supplementary material [ESM]) models: age $(p<0.05)$, region (South America) $(p<0.05), \quad$ gender $\quad($ male $) \times \mathrm{F} 3-\mathrm{F} 4 \quad($ Yes $) \times$ BLVL $(p<0.05)$, and BMI $\times$ F3-F4 (Yes) $\times$ prior response to PegIFN/R (partial responder) $(p<0.05)$. The second-order interaction term gender (male) $\times$ SVR12 (yes) $\times$ BLVL was not detected as significant.

Table 3 indicates that there was an average increase of $£ 5.86$ in total MRU-related costs for each additional year in patient's age $(p<0.05)$. With the interaction terms, a reduction of $£ 64.90$ on average total MRU-related costs was observed in prior partial responders to PegIFN/R with severe liver fibrosis compared with equivalent prior null responders across BMI values. Similarly, a reduction of $£ 49.36$ on average total MRU-related costs was identified in male patients with severe liver fibrosis compared with equivalent female patients across BLVL values. With respect to the variable region, analyses showed that total MRU-related costs tend to be reduced by $£ 223.60$ on average in South America compared with Europe $(p<0.05)$. The coefficient associated with the interaction term gender (male) $\times$ SVR12 (yes) $\times$ BLVL was not significantly different from zero in both the OLS and GLM models, and age was not detected in the GLM model.

In summary, age, region and interaction effects between gender, F3-F4, BLVL, BMI and prior response to PegIFN/ 
Table 2 Univariate predictors of total MRU-related costs

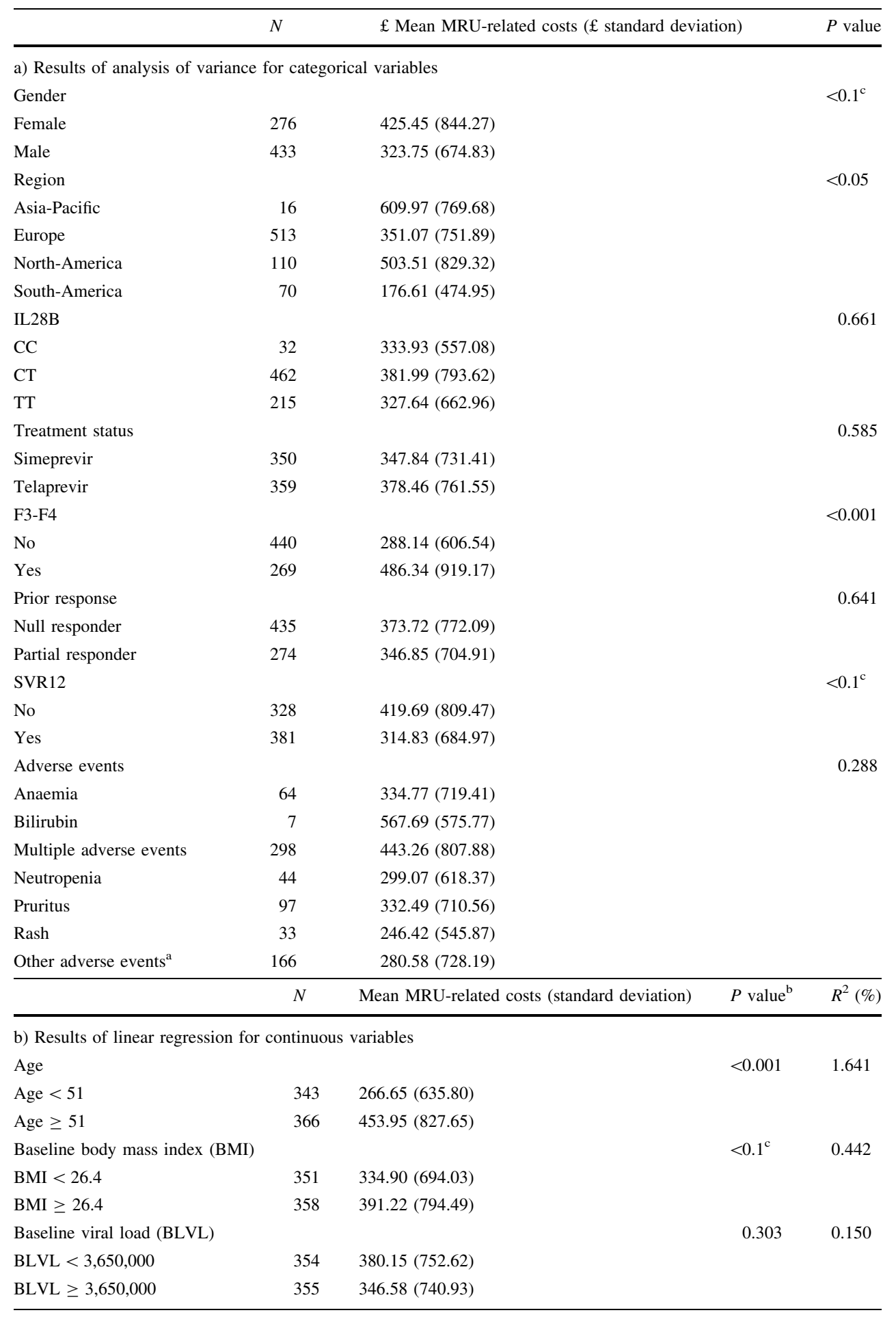

Results for continuous variables are presented in two groups split at the median value, though regression analyses were performed on the entire set of data

$B L V L$ baseline viral load in per million IU/mL, BMI body mass index in $\mathrm{kg} / \mathrm{m}^{2}, M R U$ medical resource utilization, SVR12 sustained virologic response at week 12

a Other adverse events correspond to any adverse event except anaemia, neutropenia, pruritus and rash and their combinations

${ }^{\mathrm{b}} p$ values associated with linear regression for continuous variables are those of the regression models

${ }^{c} p$ value at the borderline of the $5 \%$ significance level 
Table 3 Multivariable predictors of non-drug total MRU-related costs

\begin{tabular}{lrcc}
\hline & Estimate & Standard error & $p$ value \\
\hline Intercept & -127.702 & 295.913 & 0.666 \\
Age & 5.863 & 2.769 & $<0.05$ \\
SVR12 (yes) & 72.339 & 124.606 & 0.562 \\
Gender (male) & -90.826 & 142.968 & 0.526 \\
BLVL & -6.096 & 14.340 & 0.671 \\
F3-F4 (yes) & 201.502 & 435.020 & 0.643 \\
BMI & 6.661 & 10.128 & 0.511 \\
Prior response to PegIFN/R (partial responder) & -634.333 & 452.622 & 0.162 \\
Region (North America) & 65.032 & 81.907 & 0.428 \\
Region (South America) & -223.602 & 93.597 & $<0.05$ \\
Region (Asia-Pacific) & 196.021 & 184.983 & 0.290 \\
SVR12 (yes): gender (male) & -75.284 & 159.496 & 0.637 \\
SVR12 (yes): BLVL & 4.527 & 16.465 & 0.783 \\
Gender (male): BLVL & 31.297 & 17.297 & 0.071 \\
Gender (male): F3-F4 (yes) & 76.563 & 164.500 & 0.642 \\
BLVL: F3-F4 (yes) & 13.921 & 15.767 & 0.378 \\
F3-F4 (yes): BMI & -1.293 & 15.195 & 0.932 \\
BMI: prior response (partial response) & 21.032 & 16.796 & 0.211 \\
F3-F4 (yes): prior response (partial response) & 1869.356 & 731.496 & $<0.05$ \\
Gender (male): SVR12 (yes): BLVL & -32.336 & 20.469 & $<.115$ \\
Gender (male): F3-F4 (yes): BLVL & -49.356 & 21.578 & 26.05 \\
BMI: F3-F4 (yes): prior response (partial response) & -64.904 & 26.568 & \\
\hline A sepwise mitis & & \\
\hline
\end{tabular}

A stepwise multivariable OLS regression model was fitted to total MRU-related costs while controlling for the following factors: age, region, baseline BMI $\times$ F3-F4 $\times$ prior response to PegIFN/R, gender $\times$ F3$\mathrm{F} 4 \times \mathrm{BLVL}$ and gender $\times$ SVR12 $\times$ BLVL. Interaction terms were identified in univariate analyses as factors with linear effects on total MRU-related costs. The outcome of the present analysis was further confirmed by a generalized linear model where a gamma distribution was assumed for total MRU-related costs

$B M I$ body mass index, $B L V L$ baseline viral load, $M R U$ medical resource utilization, $O L S$ ordinary least square, PegIFN/R pegylated interferon and ribavirin, SVRI2 sustained virologic response at week 12

$R^{2}=0.088, p<0.001$

${ }^{\text {a }}$ BLVL in per million IU/mL, BMI in $\mathrm{kg} / \mathrm{m}^{2}$
$\mathrm{R}$ were identified as the most parsimonious set of predictors for total MRU-related costs. Bootstrap regression analyses also confirmed the outcome of the multivariable OLS regression model.

\subsection{Effect of the Interaction Terms}

Two approaches were used to gain insight into the interaction terms. First, simple effects (i.e. the effect of one independent variable at fixed values of the others) were calculated. For this purpose, stepwise multivariable OLS and logistic regression models were fitted to numeric and categorical variables captured in second-order interaction terms, while controlling for other patient demographics and treatment features. Age and region were also included in the assessment.

A Stepwise logistic regression analysis using gender as the dependent variable revealed significant correlations of this variable with BLVL and adverse events (Table S2, see ESM). The odds of experiencing anaemia [odds ratio (OR) $0.20 ; 95 \%$ CI $0.10-0.37 ; p<0.001]$ and multiple adverse events (OR 0.39; $95 \%$ CI $0.26-0.59 ; p<0.001$ ) was lower in male than in female patients. The likelihood of having high BLVL values was similar between genders. A borderline significant correlation was observed between age and male gender, suggesting that the male patients were a bit older than the female patients in our sample.

A stepwise multivariable logistic regression with F3-F4 as the dependent variable revealed three main factors: SVR12, age and BMI (Table S3, see ESM). Patients with severe liver fibrosis (F3-F4) had a lower chance of achieving SVR12 compared with those with mild to moderate liver fibrosis (F0-F2; OR 0.63; $95 \%$ CI $0.46-0.86 ; p<0.01)$. Likewise, higher age and higher BMI were associated with higher odds of developing severe liver fibrosis (F3-F4): OR 1.06 (95\% CI 1.04-1.07; 
$p<0.001$ ) and 1.05 (95\% CI 1.01-1.08; $p<0.05$ ), respectively.

A stepwise multivariable logistic regression with prior response to PegIFN/R as the dependent variable revealed a significant correlation with SVR12 and IL28B (Table S4, see ESM). Prior partial responders to PegIFN/R were more likely to achieve SVR12 than prior null responders (OR 2.85 ; $95 \%$ CI $2.06-3.94 ; p<0.001$ ). With an OR of 2.43 (95\% CI 1.13-5.23), prior partial responders to PegIFN/R were more likely to have the CC genotype than prior null responders to PegIFN/R $(p<0.05)$. Borderline significance was observed between age and prior partial responders to PegIFN/R, indicating that this subgroup tends to be older than the prior null responders subgroup in our sample.

The variables that proved to be strongly associated with SVR12 in a stepwise multivariable logistic regression were region, prior response to PegIFN/R, F3-F4 and adverse events (Table S5, see ESM). Results related to prior response to PegIFN/R and F3-F4 were confirmed in previous analyses (Table S3, S4, see ESM). North American treatment-experienced patients were less likely to achieve SVR12 compared with European ones (OR 0.50; 95 \% CI $0.32-0.78 ; p<0.05)$. For patients who achieved SVR12, there was an increase in the odds of having multiple adverse events (OR 2.04; $95 \%$ CI 1.36-3.06; $p<0.01$ ) or neutropenia (OR 2.74; $95 \%$ CI 1.32-5.66; $p<0.01$ ). Borderline significance was further found between SVR12 and South America, anaemia or pruritus.

Age, BMI and SVR12 were found to be significantly correlated to region in a stepwise multinomial regression analysis (Table S6, see ESM). Older patients in the selected population were more likely to be North American patients than European patients [relative risk (RR) 1.06; $95 \%$ CI $1.03-1.09 ; p<0.001]$. North American patients were also more likely to have a higher BMI than European patients (RR 1.16; $95 \%$ CI 1.11-1.22; $p<0.001$ ). Finally, North American (RR 0.48; $95 \%$ CI $0.31-0.75 ; p<0.01$ ) and South American (RR 0.59; $95 \%$ CI 0.35-0.97; $p<0.05$ ) patients were less likely to achieve SVR12 compared with European patients.

Three additional multivariable OLS regressions were conducted with age, BMI and BLVL as dependent variables. Age was significantly associated with F3-F4, BMI, BLVL and adverse events (Table S7, see ESM). Patients with severe liver fibrosis (F3-F4) were on average 5 years older than those with mild to moderate liver fibrosis (F0F2; $p<0.001)$. Similarly, strong positive correlations between age and BMI $(p<0.001)$ and age and BLVL $(p<0.05)$ were found. Patients with anaemia were on average 6 years older than those with none of the selected adverse events $(p<0.001)$. The same was true for patients experiencing multiple adverse events, who were on average
3 years older than those with none of the selected adverse events $(p<0.05)$. It was further found that BMI had a protective effect against anaemia and multiple adverse events given that patients who experienced these events had on average a lower BMI than those who did not $(p<0.05)$ (Table S8, see ESM). Finally, a strong correlation was found between BLVL and age $(p<0.05)$ and IL28B $(p<0.001)$ (Table S9, see ESM). Treatment-experienced patients with the TT genotype had, on average, two per million IU/mL BLVL lower than those with the CT genotype.

Interaction effects were also explored graphically. Figure 2 shows that male patients with severe liver fibrosis (F3-F4) had a lower BLVL than equivalent female patients. In addition, patients who were prior null responders with severe liver fibrosis had higher average BMI than equivalent patients who were partial responders. This illustrates that the direction and the magnitude of the correlations with BLVL and BMI can differ significantly by subgroup.

\subsection{Predictors of On-Treatment Adverse Events}

The association between adverse events and some of the predictors of total MRU-related costs prompted the analysis of the factors associated with on-treatment adverse events. Figure 3 shows that the occurrence of the selected adverse events was relatively higher in the TVR/PegIFN/Rtreated group than in the SMV/PegIFN/R-treated group, and therefore the absence of events was lower. Moreover, the occurrence of multiple adverse events was much higher
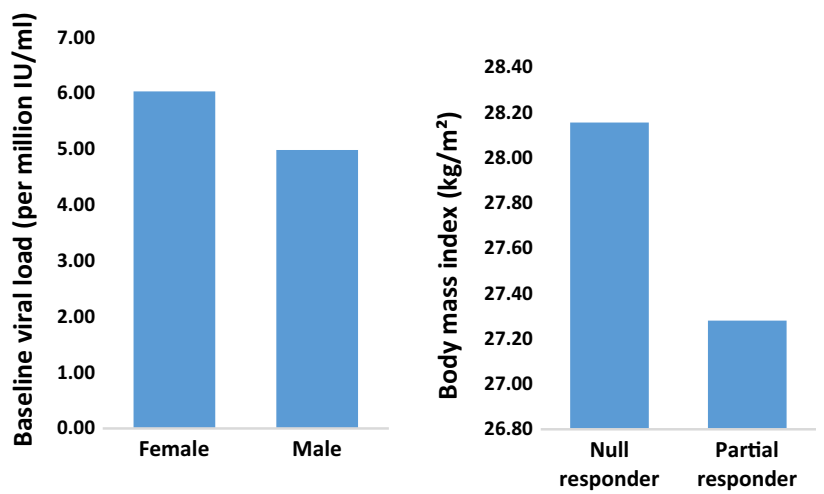

Fig. 2 Average baseline viral load in female and male patients with severe liver fibrosis and average BMI in prior non-responders to PegIFN/R with severe liver fibrosis. The graph on the left-hand side captures variables in the second-order gender $\times$ BLVL $\times$ F3-F4 interaction term. Average baseline viral load was plotted by gender levels for patients with severe liver fibrosis. The graph on the righthand side captures variables in the $\mathrm{F} 3-\mathrm{F} 4 \times \mathrm{BMI} \times$ prior response to PegIFN/R interaction term. Average baseline BMI is segmented by levels of prior response to PegIFN/R in patients with severe liver fibrosis. $B M I$ body mass index, $B L V L$ baseline viral load, PegIFN/R pegylated interferon and ribavirin 


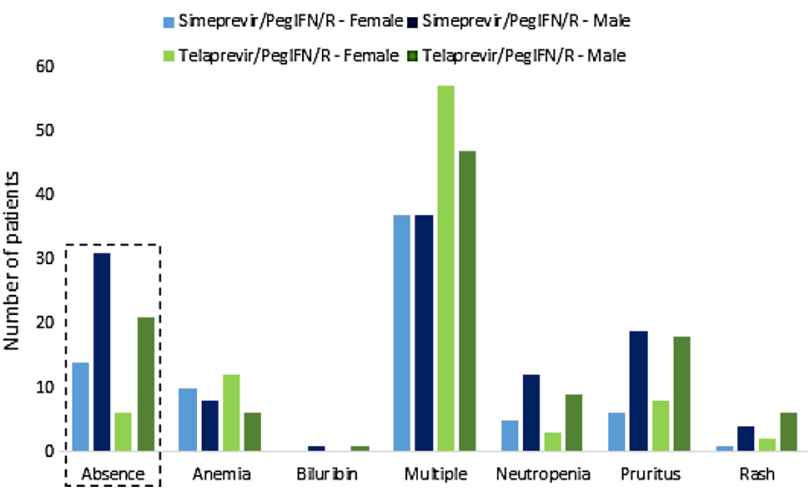

Fig. 3 Distribution of SVR12 achievers by therapy, gender and adverse events. Absence refers to patients that did not report any of the selected adverse events (i.e. anaemia, bilirubin, neutropenia, pruritus and rash). PegIFN/R pegylated interferon and ribavirin

across all treatment groups than the occurrence of any single adverse event. Multiple adverse events among female patients treated on TVR/PegIFN/R were the most commonly observed events. Further investigation of the factors associated with adverse events through multinomial regression analyses revealed that the risk of experiencing anaemia (RR 2.53; $95 \%$ CI 1.37-4.68), pruritus (RR 2.39; $95 \%$ CI 1.43-4.00), rash (RR 2.72; $95 \%$ CI 1.26-5.87) and multiple adverse events (RR 2.54; $95 \%$ CI 1.69-3.81) was significantly higher in the TVR/PegIFN/R arm than in the SMV/PegIFN/R arm (Table 4). In agreement with the simple effect analysis of the interaction terms, the correlations between age, gender, BMI, SVR12 and adverse events were further confirmed.

\subsection{Comparison of Total MRU-Related Costs Between SMV/PegIFN/R and TVR/PegIFN/R}

Previous analyses did not identify treatment status as an explanatory variable explaining the variation in total MRU-related costs. Therefore, descriptive statistics and a Wilcoxon rank sum test were used to compare MRUrelated costs between SMV/PegIFN/R and TVR/PegIFN/ $\mathrm{R}$. In line with the result from the regression analysis, no significant difference could be found for the median total MRU-related costs between the two treatment regimens (Table S10, see ESM). The same was observed for each of the 13 sub-types of medical resource. None of the patients stayed in intensive care unit. Additionally, when considering only patients with a positive cost, no difference in total MRU-related costs was detected. Overall, median total MRU-related costs (IQR) among all patients treated with SMV/PegIFN/R and all patients treated with TVR/PegIFN/R were $£ 45.22$ (£347.80) and £63.71 (£378.50), respectively.

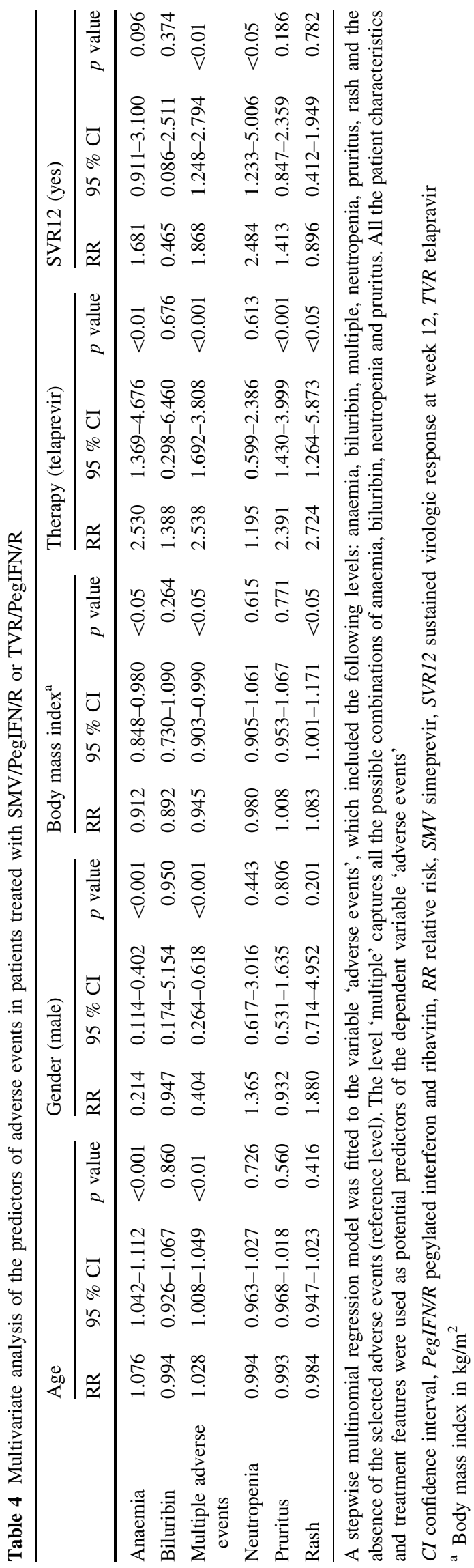




\section{Discussion}

SMV, a second generation of direct-acting antiviral HCV, led to high cure rates among patients who had previously failed with PegIFN/R. In the ATTAIN study, a pivotal trial comparing SMV/PegIFN/R with TVR/PegIFN/R, one of the first proteinase inhibitors, MRU-related data were collected to get further insights in care provision to prior non-responder patients. Among the evaluated resources was hospitalization, which accounted for the largest share of the total MRU-related costs. Savings from reduced hospitalization could potentially provide room to further optimize treatment for patients with $\mathrm{CHC}$. The present study shows how patient demographics, clinical characteristics and treatment features were associated with MRUrelated costs for treatment-experienced patients infected with CHC.

\subsection{Patient Demographics and Clinical Factors as Predictors of Total MRU-Related Costs}

Of the demographic variables included in the analysis, age was found to be significantly associated with total MRUrelated costs in univariate analyses; and with clinical factors and treatment features when evaluating interaction terms. Similarly, Gordon et al. found through a retrospective analysis of a medical and pharmacy claims database that an increase in direct healthcare costs associated with $\mathrm{CHC}$ corresponds with increasing age and disease severity [26]. Also, Razavi et al. showed higher costs in patients experiencing decompensated cirrhosis [27]. Our results further align with what was previously reported in the literature; that is, progression to cirrhosis is associated with older age and high BMI [28-31], which leads to additional costs. This finding underlines the importance of closely monitoring disease progression in older patients. Older patients are exposed to many debilitating conditions such as arthritis, depression, fatigue and anaemia; conditions with symptoms that also accompany CHC. Hence, detecting $\mathrm{CHC}$ might be challenging in older patients, given the underestimation of its true prevalence among aged patients, who are more likely to develop advanced liver disease [32, 33]. Observations from other studies reported that anaemic patients were more likely to be older, to be female and to have lower BMI [34-36].

Male gender was also identified as a predictor of total MRU-related costs when included in interaction with F3F4 and BLVL, and with SVR12 and BLVL. Multivariable logistic regression with $\mathrm{F} 3-\mathrm{F} 4$ as the dependent variable indicated that advanced fibrosis strongly affects the likelihood of achieving SVR12. In a study investigating the efficacy and safety profiles of PegIFN/R in older patients with chronic hepatitis $\mathrm{C}$, Oze et al. reported that the progression of liver fibrosis or the decrease of drug exposure concomitant with age could account for the reduction of SVR rate among these patients [37]. Using univariate and multivariable analyses to determine the factors associated with high SVR24 rates in a cohort of treatment-naïve and prior non-responder patients that received TVR/PegIFN/R, Colombo et al. identified the following predictors of SVR24: bridging fibrosis, genotype $1 \mathrm{~b}$, any previous response other than null response, baseline viral load $(<800,000 \mathrm{IU} / \mathrm{mL})$ and alpha fetoprotein concentration $(<10 \mu \mathrm{g} / \mathrm{L})[38,39]$. Our results confirm that patients who achieve SVR12 consume less medical resources; an effect, however, dependent on BLVL and liver fibrosis stage in treatment-experienced patients. This probably explains why the interaction term gender $\times$ SVR $12 \times$ BLVL was no longer significant in multivariable regression analysis upon the inclusion of the interaction term gender $\times$ F3F4 $\times$ BLVL. F3-F4 is likely a stronger predictor than SVR12 on the MRU-related costs, and diminished its effect. This is clear from the strong correlation between F3-F4 and SVR12.

In the currently studied population, total MRU-related costs were significantly lower in male patients with F3-F4 METAVIR score and low BLVL compared with their equivalent female patients with high BLVL. Consequently, it is important to monitor liver disease severity and BLVL together and not independently as these parameters can reduce total MRU-related costs significantly when patients with $\mathrm{CHC}$ are treated early. Such an outcome has substantial implications when evaluating, for example, treatment strategies against $\mathrm{HCV}$ before and after liver transplantation in patients with advanced liver fibrosis [40]. It has been reported that spontaneous clearance of $\mathrm{HCV}$ after transplantation is rare while reinfection of the allograft is common; therefore reducing the prognosis of these patients [41-44]. Adopting antiviral drugs that could eradicate the virus from the bloodstream and reduce the risk of reinfection of the allograft could then potentially generate substantial savings in MRU-related costs and improve the condition of the patient.

The second interaction effect on total MRU-related costs was $\mathrm{BMI} \times \mathrm{F} 3-\mathrm{F} 4 \times$ prior response to PegIFN/R. This result confirms the positive correlation between high BMI, the pathogenesis of steatosis and fibrosis, and the fact that high BMI has been reported to be an independent risk factor for non-response to antiviral therapy [45-47]. It might not be surprising to observe lower total MRU-related costs in prior partial responders than in prior null responders to PegIFN/R with severe liver fibrosis. This is the consequence of (1) higher BMI and older age being associated with severe liver fibrosis, (2) patients with severe liver fibrosis being less likely to achieve SVR than those 
with mild to moderate liver fibrosis and (3) in the current setting, the average BMI being lower in prior partial responders with F3-F4 METAVIR stage than their equivalent null responders to PegIFN/R. Loss of weight should thus be considered when treating prior non-responders to PegIFN/R with advanced liver fibrosis.

Region was further detected as a predictor of total MRU-related costs. South American patients showed lower total MRU-related costs than European patients. Number of visits to a physiotherapist and associated costs were higher among South American than European patients whereas the number of visits to the primary care physician were lower among South American patients compared with European patients (data not shown). The higher unit cost for primary-care physician visits than for physiotherapist visits explains why lower MRU-related costs were observed among South American patients. This indicates that patients' access to specialists is to be taken into consideration to manage non-drug MRU-related costs.

This study has also identified factors predictive of adverse events in prior non-responders to PegIFN/R treated with SMV/PegIFN/R and TVR/PegIFN/R. Female gender, older age and low BMI were predictors of anaemia; an observation confirmed in previous publications and irrespective of naïve and experienced treatment populations [34-36, 48-50]. In a review article, Boccaccio and Bruno further emphasized the higher risk of anaemia in older patients treated with TVR/PegIFN/R [51]. With respect to rash, Smith et al., in a retrospective observational study, indicated that actual body weight and BMI appear to be related to rash development in patients treated with TVR/ PegIFN/R, and suggested that this finding may have implications in the treatment of HCV with SMV/PegIFN/R [52]. Roujeau et al. further reported that the incidence of TVR-related dermatitis was significantly higher with age $>45$ years, BMI $<30 \mathrm{~kg} / \mathrm{m}^{2}$, White race, and if receiving therapy for $\mathrm{HCV}$ for the first time [53]. In a meta-analysis performed by the Canadian Agency for Drugs and Technologies in Health, no consistent increased risk of rash was found between BCP or TVR and SMV in treatment-experienced patients [54]. The ATTAIN head-to-head trial further confirmed the superior safety profile of SMV/ PegIFN/R compared with TVR/PegIFN/R for anaemia, pruritus and rash [9]; therefore limiting the extrapolation of the risk profile of TVR/PegIFN/R to SMV/PegIFN/R as suggested by Smith et al. Finally, this study shows also that SVR12 achievers were more likely to be exposed to multiple adverse events and neutropenia. It is well acknowledged that interferon (IFN) therapy causes a decrease in neutrophil counts, as a result of bone marrow suppression [55]. Retreatment of prior non-responders to PegIFN/R with an IFN-based regimen increases their exposure to IFN, potentially elevating the risk of neutropenia.

\subsection{Differences Between SMV/PegIFN/R and TVR/PegIFN/R}

SMV/PegIFN/R was found to have a comparable impact on total MRU-related costs to TVR/PegIFN/R, despite its improved safety profile. This similarity in patterns of MRU-related costs between the two treatment options could be explained by factors other than the adverse events, including the stronger impact of disease severity on costs, the comparable efficacy between treatments, or by the fact that potentially not all the medical services were captured in this study.

Regarding the latter point, it should be noted that renal dysfunction (not captured in our data) is recognized as a side effect of TVR/PegIFN/R therapy [51, 56]. Identified factors associated with renal impairment when treated with TVR/PegIFN/R included advanced age and low initial haemoglobin levels. On the other hand, creatinine clearance was not found to influence pharmacokinetic parameters of SMV/PegIFN/R in pharmacokinetic studies in HCV-infected patients with mild to moderate renal impairment treated with SMV/PegIFN/R [57]. Besides the care services captured in the present study, an assessment of resources required for the management of renal complications could thus have been of interest. Not only that, but also visits to dermatologists would have likely had an impact on MRU-related costs given that patients treated with TVR/PegIFN/R were exposed to a higher risk of pruritus and rash. These resources were not specifically captured in the clinical trial and thus were not included in the analyses described above.

Though well recognized as a major component of the overall care costs, the costs of drugs were not analysed in the current study. The rationale behind this approach was that there was no substantial difference in co-medication intake (defined as the treatment length multiplied by the dose frequency) and co-medication consumption (defined as the treatment length multiplied by the dose frequency and the unit of the dosage form-tablet/capsule or suspension) between the two treatment arms (data not shown). Moreover, the main costs would be related to the drugs acting directly against $\mathrm{CHC}$, and these were also not different by treatment arm due to the similar price levels of SMV and TVR. Finally, this study primarily aims at identifying patient and treatment characteristics that would influence non-drug MRU-related costs, so that these could be better managed via the implementation of efficient care programmes as alternative routes so as to optimise clinical management and generate additional savings. Focusing on utilization management approaches has also been proposed for the three primary disease areas that account for the majority of the specialty drug spending, including autoimmune disorders, multiple sclerosis and cancer [58-60]. 
The comparison of the level of MRU-related costs with data from the literature was not evident given the lack of data on MRU-related costs for prior non-responders to PegIFN/R. However, our results aligned with the nondrug-related costs of $£ 806$ reported by Backx et al. in retreating non-SVR achievers with PegIFN/R; though the following medical resources were captured: outpatient clinic visits, HCV RNA tests, liver-related imaging, daycase visits (e.g. endoscopy, liver biopsy) and inpatient hospital stays [17]. In another study, the costs in Germany were captured retrospectively. Stahmeyer et al. investigated the following medical services: doctor visits, diagnostic and laboratory tests, pharmaceuticals and inpatient care [13]. The mean outpatient cost (including diagnostic procedures and excluding pharmaceuticals) was $€ 1686$. The average inpatient cost was estimated at $€ 1293$. Lastly, a Belgian study by Nevens et al. evaluated direct medical costs on the basis of hospitalizations, day-clinic visits, surgery/interventions, physician visits, and diagnostic tests and drug use over a 3-year follow-up period [61]. Direct medical costs (hospitalization and ambulatory care) were estimated at $€ 3609$ for patients with mild CHC. Likewise, patients with moderate disease and cirrhosis without varices (abnormally dilated vessels) and those with cirrhosis and varices had an average non-drugrelated cost of $€ 3150$ and of $€ 6098$, respectively. Conclusively, the direct comparison of MRU and MRU-related costs across these studies is challenging given selected patient demographic and clinical characteristics, study designs, treatment status (triple or dual PegIFN/R regimens), type of response (treatment naïve or experienced), healthcare provision and cost structures. Moreover, in these studies, antiviral therapy included exclusively PegIFN/R and thus did not include the effect of a protease inhibitor like this study did.

Finally, this study showed that the adverse events were significantly different between treatment arms and are therefore relevant to consider when selecting between treatment options for prior non-responders to PegIFN/R. Owing to the more favourable safety profile [9], the similar drug price levels and the comparable MRU-related non-drug costs of SMV/PegIFN/R as compared with TVR/PegIFN/R, SMV/PegIFN/R could be suggested as an alternative to TVR/PegIFN/R for treatment-experienced patients, especially in national settings and in some subgroups of patients where an IFN-based regimen might still be used to limit the higher costs associated with an IFN-free regimen; and/or when justifications of the risk/ benefit profile of antiviral combinations is required to support the medically accepted indication and reimbursement.

\subsection{Strengths, Limitations and Future Directions}

The multivariable OLS regression analysis predicted $9 \%$ of the variance in total MRU-related costs, indicating the independent role played by age and interaction effects of gender, F3-F4 and BLVL; and of BMI, F3-F4 and prior response to PegIFN/R on total MRU-related costs patterns. Additional research would be required to identify other variables that could increase the predictive power of MRU and related non-drug costs in treatment-experienced patients.

As previously mentioned, there may be additional medical services, patient characteristics and treatment features that were not captured in the present study and that would have predicted substantial variance in total MRUrelated costs. Moreover, the current study does not capture medication costs and laboratory tests but only the costs associated with providing specific outpatient care and hospitalization. Indirect costs were further not analysed.

The results from this study have strong implications for policy makers, especially with the arrival of new antiviral drugs and their combinations from late 2014 and beyond. New regimens have been explored including the SMV/SOF (sofosbuvir) combination, which was efficacious in null responders and treatment-naïve patients across all levels of liver fibrosis, as well as in patients with the Q80K polymorphism [62]. SMV/SOF has further demonstrated efficacy in patients with previous protease inhibitor failure, despite retreatment comprising a protease inhibitor [63]. Prior non-responders are traditionally considered as a difficult group to treat and, because treatment for $\mathrm{CHC}$ began as early as the 1990s, many of those living with HCV today are considered treatment-experienced patients [64]. With cure rates higher than $90 \%$, the difference between new regimens and their effective and safe combination (including decreased drug interactions, adverse events, side effects and improved dosing regimens) will likely lie in the ease of use (e.g. reduced pill burden), the shortened treatment duration, costs and the high barrier to HCV resistance. Access to care will then be an increasing point of focus; hence the importance for policymakers to implement early-stage curative strategies to avoid disease progression and cost burden.

This study advocates the simultaneous monitoring of liver disease severity and BLVL in special and difficult-totreat populations based on age, gender and BMI. Further research is required to comprehend how patient demographics, clinical characteristics and treatment features of IFN-free regimens in staged treatment alternatives would improve non-drug-related savings and efficiency in care delivery for PegIFN/R and proteinase inhibitor-experienced patients. 
This study also has implications in national settings and in some subgroups of patients where an IFN-based regimen might still be used to limit the higher costs associated with an IFN-free regimen, and/or when justifications of the risk/ benefit profile of antiviral combinations is required to support the medically accepted indication and reimbursement.

\subsection{Conclusion and Clinical Implications}

In this population of prior non-responders to PegIFN/R, higher age and interaction effects between gender, F3-F4 METAVIR score and high BLVL; and between high BMI, F3-F4 METAVIR stage and prior response to PegIFN/R were found to be significantly associated with higher MRUrelated costs. In addition, F3-F4 METAVIR score was a stronger predictor for costs than SVR12 in prior partial and prior null responders to PegIFN/R. This suggests that by starting treatment early, before $\mathrm{CHC}$ progresses, significant treatment costs can be avoided. Moreover, weight loss should further be encouraged in patients with $\mathrm{CHC}$ with a high BMI to avoid additional costs.

Although there was no significant difference in costs between treatment arms, SMV/PegIFN/R was associated with a significantly lower likelihood of having adverse events than TVR/PegIFN/R. This indicates that adverse events are an important parameter to take into consideration for the choice of treatment.

Acknowledgments The authors acknowledge Chris Corbett for his advice on the manuscript.

\section{Compliance with Ethical Standards}

Conflict of interest The authors E. I. H. A. and J. K. declare no conflict of interest. U. S. and G. W. are employees of Janssen Pharmaceutica. All the analyses were performed by E. I. H. A. The manuscript was written by E. I. H. A. and J. K. U. S. and G. W. provided access to the simeprevir trial data and comments on the manuscript. The study guarantor is U. S. All authors approved the final version of the article. The authors have no other conflicts of interest that are directly relevant to the content of this article.

Funding sources This study was sponsored by Janssen Pharmaceutica, which holds the market authorization of simeprevir.

Open Access This article is distributed under the terms of the Creative Commons Attribution-NonCommercial 4.0 International License (http://creativecommons.org/licenses/by-nc/4.0/), which permits any noncommercial use, distribution, and reproduction in any medium, provided you give appropriate credit to the original author(s) and the source, provide a link to the Creative Commons license, and indicate if changes were made.

\section{References}

1. Lavanchy D. Evolving epidemiology of hepatitis $\mathrm{C}$ virus. Clin Microbiol Infect. 2011;17(2):107-15.
2. Chak E, Talal AH, Sherman KE, Schiff ER, Saab S. Hepatitis C virus infection in USA: an estimate of true prevalence. Liver Int. 2011;31(8):1090-101.

3. Jemal A, Bray F, Center MM, Ferlay J, Ward E, Forman D. Global cancer statistics. CA Cancer J Clin. 2011;61(2):69-90.

4. Blachier M, Leleu H, Peck-Radosavljevic M, Valla DC, Roudot-Thoraval F. The burden of liver disease in Europe: a review of available epidemiological data. J Hepatol. 2013;58(3): 593-608.

5. Freeman AJ, Dore GJ, Law MG, Thorpe M, Von OJ, Lloyd AR, et al. Estimating progression to cirrhosis in chronic hepatitis $\mathrm{C}$ virus infection. Hepatology. 2001;34(4 Pt 1):809-16.

6. Fried MW, Buti M, Dore GJ, Flisiak R, Ferenci P, Jacobson I, et al. Once-daily simeprevir (TMC435) with pegylated interferon and ribavirin in treatment-naive genotype 1 hepatitis $\mathrm{C}$ : the randomized PILLAR study. Hepatology. 2013;58(6):1918-29.

7. Jacobson IM, Dore GJ, Foster GR, Fried MW, Radu M, Rafalsky $\mathrm{VV}$, et al. Simeprevir with pegylated interferon alfa $2 \mathrm{a}$ plus ribavirin in treatment-naive patients with chronic hepatitis $\mathrm{C}$ virus genotype 1 infection (QUEST-1): a phase 3, randomised, doubleblind, placebo-controlled trial. Lancet. 2014;384(9941):403-13.

8. Manns M, Marcellin P, Poordad F, de Araujo ES, Buti M, Horsmans Y, et al. Simeprevir with pegylated interferon alfa 2a or $2 \mathrm{~b}$ plus ribavirin in treatment-naive patients with chronic hepatitis $\mathrm{C}$ virus genotype 1 infection (QUEST-2): a randomised, double-blind, placebo-controlled phase 3 trial. Lancet. 2014; 384(9941):414-26.

9. Reddy KR, Zeuzem S, Zoulim F, Weiland O, Horban A, Stanciu $\mathrm{C}$, et al. Simeprevir versus telaprevir with peginterferon and ribavirin in previous null or partial responders with chronic hepatitis $\mathrm{C}$ virus genotype 1 infection (ATTAIN): a randomised, double-blind, non-inferiority phase 3 trial. Lancet Infect Dis. 2015;15(1):27-35.

10. Blatt C, da Cunha Bernardo N, Rosa J, Bagatini F, Alexandre R, Neto G, et al. An estimate of the cost of hepatitis $C$ treatment for the Brazilian health system. Value Health Reg Issues. 2012;1:129-35.

11. Sánchez-Ávila J. Cost efficacy and cost-benefit of treatment of hepatitis C. Ann Hepatol. 2006;5(Suppl. 1):S69-73.

12. Stahmeyer JT, Rossol S, Bert F, Abdelfattah AM, Mauss S, Heyne R, et al. Epidemiology, treatment outcomes and costs of treating hepatitis $\mathrm{C}$ in routine care-results from a large multicenter trial. Value Health. 2013;16(7):A341-2.

13. Stahmeyer JT, Rossol S, Bert F, Antoni C, Demir M, Hinrichsen $\mathrm{H}$, et al. Cost of treating hepatitis $\mathrm{C}$ in Germany: a retrospective multicenter analysis. Eur J Gastroenterol Hepatol. 2014;26(11): 1278-85.

14. Su J, Brook RA, Kleinman NL, Corey-Lisle P. The impact of hepatitis $\mathrm{C}$ virus infection on work absence, productivity, and healthcare benefit costs. Hepatology. 2010;52(2):436-42.

15. Chronic conditions: making the case for ongoing care. Partnership for Solutions. Johns Hopkins University for The Robert Wood Johnson Foundation; 2002.

16. Yu W, Ravelo A, Wagner TH, Phibbs CS, Bhandari A, Chen S, et al. Prevalence and costs of chronic conditions in the VA health care system. Med Care Res Rev. 2003;60(3 Suppl):146S-67S.

17. Backx M, Lewszuk A, White JR, Cole J, Sreedharan A, van Sanden S, et al. The cost of treatment failure: resource use and costs incurred by hepatitis $\mathrm{C}$ virus genotype 1-infected patients who do or do not achieve sustained virological response to therapy. J Viral Hepat. 2014;21(3):208-15.

18. Manos MM, Darbinian J, Rubin J, Ray GT, Shvachko V, Denis $\mathrm{B}$, et al. The effect of hepatitis $\mathrm{C}$ treatment response on medical costs: a longitudinal analysis in an integrated care setting. J Manag Care Pharm. 2013;19(6):438-47. 
19. Department of Health. Reference costs 2011-12. National Health Services; 2012.

20. Curtis L, Netten A. Unit costs of health and social care 2008. Canterbury: The University of Kent, Personal Social Services Research Unit; 2008.

21. Curtis L. Unit costs of health and social care 2007. Canterbury: University of Kent, Personal Social Services Research Unit; 2007.

22. Office for National Statistics. Consumer Price Inflation Reference Tables, March 2013. UK Statistics Authority 2013. http://www.ons. gov.uk/ons/rel/cpi/consumer-price-indices/march-2013/consumerprice-inflation-reference-tables.xls. Accessed 16 May 2013.

23. Thompson SG, Barber JA. How should cost data in pragmatic randomised trials be analysed? BMJ. 2000;320(7243):1197-200.

24. Efron B, Tibshirani RJ. An introduction to the bootstrap. New York: CRC press; 1993.

25. Daggy JK, Thomas J III, Craig BA. Modeling correlated healthcare costs. Expert Rev Pharmacoecon Outcomes Res. 2011;11(1):101-11.

26. Gordon SC, Pockros PJ, Terrault NA, Hoop RS, Buikema A, Nerenz D, et al. Impact of disease severity on healthcare costs in patients with chronic hepatitis $\mathrm{C}(\mathrm{CHC})$ virus infection. Hepatology. 2012;56(5):1651-60.

27. Razavi H, Elkhoury AC, Elbasha E, Estes C, Pasini K, Poynard T, et al. Chronic hepatitis $\mathrm{C}$ virus (HCV) disease burden and cost in the United States. Hepatology. 2013;57(6):2164-70.

28. Kirk GD, Mehta SH, Astemborski J, Galai N, Washington J, Higgins Y, et al. HIV, age, and the severity of hepatitis $\mathrm{C}$ virusrelated liver disease: a cohort study. Ann Intern Med. 2013;158(9):658-66.

29. Taura N, Yatsuhashi H, Hamasaki K, Nakao K, Daikoku M, Ueki $\mathrm{T}$, et al. Increasing hepatitis $\mathrm{C}$ virus-associated hepatocellular carcinoma mortality and aging: long term trends in Japan. Hepatol Res. 2006;34(2):130-4.

30. Poynard T, Bedossa P, Opolon P. Natural history of liver fibrosis progression in patients with chronic hepatitis C. The OBSVIRC, METAVIR, CLINIVIR, and DOSVIRC groups. Lancet. 1997;349(9055):825-32.

31. Ghany MG, Strader DB, Thomas DL, Seeff LB. Diagnosis, management, and treatment of hepatitis C: an update. Hepatology. 2009;49(4):1335-74.

32. Gramenzi A, Conti F, Felline F, Cursaro C, Riili A, Salerno M, et al. Hepatitis C Virus-related chronic liver disease in elderly patients: an Italian cross-sectional study. J Viral Hepat. 2010;17(5):360-6.

33. Kim HI, Kim IH, Jeon BJ, Lee S, Kim SH, Kim SW, et al. Treatment response and tolerability of pegylated interferon-alpha plus ribavirin combination therapy in elderly patients $(>/=65$ years) with chronic hepatitis C in Korea. Hepat Mon. 2012;12(7):430-6.

34. Aapro MS, Cella D, Zagari M. Age, anemia, and fatigue. Semin Oncol. 2002;29(3 Suppl 8):55-9.

35. Dan AA, Martin LM, Crone C, Ong JP, Farmer DW, Wise T, et al. Depression, anemia and health-related quality of life in chronic hepatitis C. J Hepatol. 2006;44(3):491-8.

36. Bichoupan K, Schwartz JM, Martel-Laferriere V, Giannattasio ER, Marfo K, Odin JA, et al. Effect of fibrosis on adverse events in patients with hepatitis $\mathrm{C}$ treated with telaprevir. Aliment Pharmacol Ther. 2014;39(2):209-16.

37. Oze T, Hiramatsu N, Yakushijin T, Mochizuki K, Oshita M, Hagiwara $\mathrm{H}$, et al. Indications and limitations for aged patients with chronic hepatitis $\mathrm{C}$ in pegylated interferon alfa-2b plus ribavirin combination therapy. J Hepatol. 2011;54(4):604-11.

38. Colombo M, Strasser SI, Moreno C, Ferreira PA, Urbanek P, Fernandez I, et al. Sustained virological response with telaprevir in 1078 patients with advanced hepatitis C: the International Telaprevir Access Program. J Hepatol. 2014;61(5):976-83.
39. Sarrazin C, Susser S, Doehring A, Lange CM, Muller T, Schlecker $\mathrm{C}$, et al. Importance of IL28B gene polymorphisms in hepatitis $\mathrm{C}$ virus genotype 2 and 3 infected patients. J Hepatol. 2011;54(3):415-21.

40. Sugawara Y, Tamura S, Kokudo N. Antiviral treatment for hepatitis $\mathrm{C}$ virus infection after liver transplantation. Hepat Res Treat. 2010;2010:475746.

41. Dale CH, Burns P, McCutcheon M, Hernandez-Alejandro R, Marotta PJ. Spontaneous clearance of hepatitis $\mathrm{C}$ after liver and renal transplantation. Can J Gastroenterol. 2009;23(4):265-7.

42. Samonakis DN, Cholongitas E, Triantos CK, Griffiths P, Dhillon AP, Thalheimer U, et al. Sustained, spontaneous disappearance of serum HCV-RNA under immunosuppression after liver transplantation for $\mathrm{HCV}$ cirrhosis. J Hepatol. 2005; 43(6):1091-3.

43. Wiesner RH, Sorrell M, Villamil F. Report of the first International Liver Transplantation Society expert panel consensus conference on liver transplantation and hepatitis C. Liver Transpl. 2003;9(11):S1-9.

44. Berenguer M, Lopez-Labrador FX, Wright TL. Hepatitis C and liver transplantation. J Hepatol. 2001;35(5):666-78.

45. Hourigan LF, Macdonald GA, Purdie D, Whitehall VH, Shorthouse C, Clouston A, et al. Fibrosis in chronic hepatitis C correlates significantly with body mass index and steatosis. Hepatology. 1999;29(4):1215-9.

46. Bressler BL, Guindi M, Tomlinson G, Heathcote J. High body mass index is an independent risk factor for nonresponse to antiviral treatment in chronic hepatitis C. Hepatology. 2003; 38(3):639-44.

47. Hickman IJ, Powell EE, Prins JB, Clouston AD, Ash S, Purdie $\mathrm{DM}$, et al. In overweight patients with chronic hepatitis C, circulating insulin is associated with hepatic fibrosis: implications for therapy. J Hepatol. 2003;39(6):1042-8.

48. Singh G, Issa D, Sedki E, Hanouneh I, Lopez R, Zein N, et al. Anemia in patients with chronic hepatitis $\mathrm{C}$ infection during triple therapy with telaprevir or boceprevir-a controlled study. J Antivir Antiretrovir. 2013;5:173-7.

49. Colombo M, Fernandez I, Abdurakhmanov D, Ferreira PA, Strasser SI, Urbanek P, et al. Safety and on-treatment efficacy of telaprevir: the early access programme for patients with advanced hepatitis C. Gut. 2014;63(7):1150-8.

50. Akpo EI, Cerri K, Kleintjens J. Predicting the impact of adverse events and treatment duration on medical resource utilizationrelated costs in hepatitis $\mathrm{C}$ genotype 1 treatment-naive patients receiving antiviral therapy. Pharmacoeconomics. 2015;33(4): 409-22.

51. Boccaccio V, Bruno S. Optimal management of patients with chronic hepatitis C and comorbidities. Liver Int. 2015;35(Suppl 1):35-43

52. Smith MA, Johnson HJ, Chopra KB, Dunn MA, Ulrich AM, Mohammad RA. Incidence and management of rash in telaprevirtreated patients. Ann Pharmacother. 2014;48(9):1166-71.

53. Roujeau JC, Mockenhaupt M, Tahan SR, Henshaw J, Martin EC, Harding M, et al. Telaprevir-related dermatitis. JAMA Dermatol. 2013;149(2):152-8.

54. Canadian Agency for Drugs and Technology in Health. CADTH therapeutic review. Direct-acting antiviral agents for chronic hepatitis C Genotype 1. 2014. http://www.cadth.ca/media/pdf/ TR0007_HepC_RecsReport_e.pdf. Accessed 1 Mar 2015.

55. Soza A, Everhart JE, Ghany MG, Doo E, Heller T, Promrat K, et al. Neutropenia during combination therapy of interferon alfa and ribavirin for chronic hepatitis C. Hepatology. 2002;36(5): 1273-9.

56. Karino T, Ozeki I, Hige S, Kimura M, Arakawa T, Nakajima T, et al. Telaprevir impairs renal function and increases blood ribavirin concentration during telaprevir/pegylated interferon/ 
ribavirin therapy for chronic hepatitis C. J Viral Hepat. 2014;21(5):341-7.

57. Mauss S, Buti M, Moreno C, Foster GR, DeMasi R, Baldini A et al. Renal function in HCV genotype 1-infected treatment-naïve patients receiving simeprevir in combination with Peg-IFN and ribavirin: a post-hoc analysis. J.Viral Hepat. 2014;21.

58. Kenney JT Jr. Payers' management of oncology drugs: opportunities and challenges. Am Health Drug Benefits. 2014;7(3):123-4.

59. DaCosta BS, Nash SE, Mytelka D, Bowman L, Teitelbaum A. Healthcare costs, treatment patterns, and resource utilization among pancreatic cancer patients in a managed care population. J Med Econ. 2013;16(12):1379-86.

60. Miller MK, Lee JH, Blanc PD, Pasta DJ, Gujrathi S, Barron H, et al. TENOR risk score predicts healthcare in adults with severe or difficult-to-treat asthma. Eur Respir J. 2006;28(6):1145-55.
61. Nevens F, Colle I, Michielsen P, Robaeys G, Moreno C, Caekelbergh K, et al. Resource use and cost of hepatitis C-related care. Eur J Gastroenterol Hepatol. 2012;24(10):1191-8.

62. Lawitz E, Sulkowski MS, Ghalib R, Rodriguez-Torres M, Younossi ZM, Corregidor A, et al. Simeprevir plus sofosbuvir, with or without ribavirin, to treat chronic infection with hepatitis $\mathrm{C}$ virus genotype 1 in non-responders to pegylated interferon and ribavirin and treatment-naive patients: the COSMOS randomised study. Lancet. 2014;384(9956):1756-65.

63. Dusheiko G. Controlling hepatitis C with simeprevir. Lancet Infect Dis. 2015;15(1):2-4.

64. Welch NM, Jensen DM. Pegylated interferon based therapy with second-wave direct-acting antivirals in genotype 1 chronic hepatitis C. Liver Int. 2015;35(Suppl. 1):11-7. 